\title{
Sensor packaging
}

\section{An Ultrahigh Vacuum Packaging Process Demonstrating Over 2 Million Q-Factor in MEMS Vibratory Gyroscopes}

\author{
Mohammad H. Asadian* (i) , Sina Askari*, and Andrei M. Shkel** (]) \\ Microsystems Laboratory, Department of Mechanical and Aerospace Engineering, University of California, Irvine, CA 92697 USA \\ * Student Member, IEEE \\ ***Fellow, IEEE
}

Manuscript received August 14, 2017; revised September 15, 2017; accepted October 6, 2017. Date of publication October 11, 2017; date of current version November 2, 2017.

\begin{abstract}
Micro resonators and vibratory gyroscopes could reach their fundamental thermo-elastic damping (TED) limit in an ultravacuum condition where the viscous damping is eliminated. This article presents a reliable and repeatable high vacuum sealing process as a standalone approach for performance evaluation of dynamic micro devices. We demonstrated a micro-Torr vacuum level in a ceramic leadless chip carrier package and achieved the Q-factor over 2 million on a quad mass gyroscope, approaching the TED limit of the device by effectively eliminating the viscous damping. The long-term vacuum stability was characterized over 1 year and demonstrated that the Q-factor does not degrade over time and even continues improving. The long-term ultrahigh vacuum condition is enabled by surface desorption prior to sealing, pumping of residual gases by passive getters, and defect-free solder reflow in the sealing area. The experimental results confirmed the reliability and repeatability of the proposed sealing procedure, which can be adapted for other Micro Electro-Mechanical Systems (MEMS) with ultrahigh vacuum requirements.
\end{abstract}

Index Terms—Sensor packaging, vacuum packaging, inertial sensors, gyroscopes, long-term stability, quality factor.

\section{INTRODUCTION}

The Q-factor of micromachined Coriolis Vibratory Gyroscopes (CVGs) primarily depends on air damping, anchor losses, asymmetry of structures, material internal losses, surface losses, electrical losses, and Thermo-Elastic Damping (TED). The weakest link among the energy dissipation mechanisms limits the overall Q-factor of a resonant Micro Electro-Mechanical Systems (MEMS), [1]. In MEMS devices, because of the large surface-to-volume ratio and small spacing between static and moving structures, viscous damping is the dominant energy dissipation mechanism. Therefore, high Q and high-performance sensors, such as Disk Resonator Gyroscopes (DRG) [2], silicon tuning fork resonators and gyroscopes [3], [4], and fused silica 3-D shell resonators [5], [6], must operate at high vacuum level for higher sensitivity. Operating in a vacuum chamber or using on-board vacuum techniques, discrete vacuum packaging [2], [4] and wafer-level packaging [7]-[9] are the approaches to reduce the viscous damping in dynamic MEMS devices.

A high vacuum stability is also required to avoid performance drift in the long run. The pressure inside a sealed cavity evolves during the lifetime of the device. The main sources of vacuum degradation are the desorption of gas from the internal surfaces (outgassing) and the leakage in the sealing, [10], [11]. The outgassing is an unavoidable phenomenon and, from the thermodynamic point of view, is a result of a complex gas-solid interaction inside a hermetically sealed package, [12]. The outgassing rate highly depends on the initial concentration of physically adsorbed gas molecules on the solid surface. Thus, selecting vacuum compatible and low outgassing materials for fabrication

Corresponding author: M. H. Asadian (e-mail: asadianm@uci.edu).

Associate Editor: D. Elata.

Digital Object Identifier 10.1109/LSENS.2017.2762287 and packaging, and including the pre-baking step is shown to reduce the outgassing rate. However, the pressure, caused by outgassing in the long run, can only be compensated by integration and activation of getter inside the sealed cavities. An effective activation of getter increases its pumping efficiency, resulting in a long-term vacuum stability. In addition, defects in the sealing area, such as air voids or micro-cracks on interfaces, would allow gas molecules entering the cavity, evolve the pressure during lifetime of the device. A defect-free sealing can be obtained by appropriate treatment of packaging components and suitable parameters selection of the sealing process.

In a successful MEMS vacuum sealing, it is critical to adjust the temperature profile of pre-bake, getter activation, and solder reflow processes to achieve a long-term vacuum stability. A high-level vacuum sealing requires a long pre-baking of components to sufficiently reduce the concentration of trapped gases and water vapor molecules from the internal surfaces. The number of remained molecules depends on the temperature and duration of the pre-baking process. Integration of non-evaporable getter enables pumping of residual gas molecules, maintaining a stable vacuum level during lifetime of the device. This paper presents a procedure for package-level vacuum sealing of silicon micromachined resonant gyroscopes emphasizing restrictions in materials and temperature compatibility, thus providing the complete information for adapting the process to other MEMS devices.

\section{VACUUM PACKAGING PROCESS}

\section{A. Material Selection and Handling}

In this work, Ceramic Leadless Chip Carrier packages, Kovar lids with a thin film deposited getter (PaGeLid SAES), and Gold-Tin (AuSn 80/20) eutectic alloy solder preforms were used for vacuum packaging 


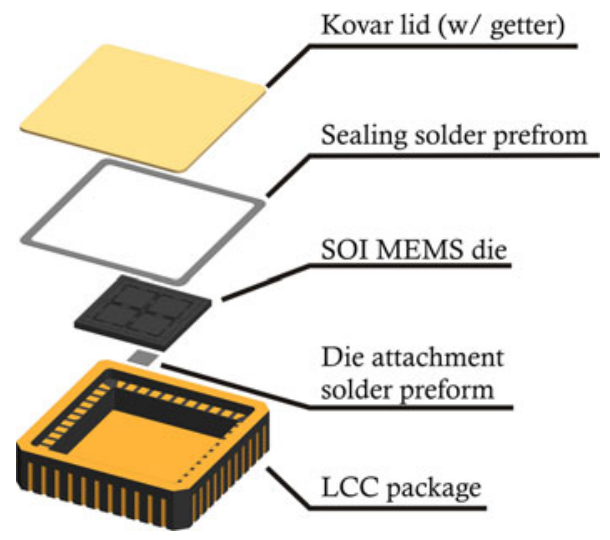

Fig. 1. Exploded view of materials used for vacuum sealing process.

of Silicon-on-Insulator (SOI) Quad Mass Gyroscope (QMG) sensors. Fig. 1 shows an exploded view of all the parts of a vacuum packaged MEMS sensor. A $2 \mu \mathrm{m}$ thin film PaGeLid getter was deposited on the inner surface of the Kovar lids, forming the total getter area of $140 \mathrm{~mm}^{2}$. All the packaging parts were stored in desiccating cabinets prior to the sealing process to reduce any surface adsorption. The cleanliness and vacuum compatibility of materials used for the device packaging dramatically influence the yield of vacuum packaging process.

\section{B. Eutectic Die Attachment}

LCC packages were initially baked at $400{ }^{\circ} \mathrm{C}$ for 7 to 10 hours in high vacuum $\left(<10^{-5}\right.$ Torr $)$ to remove the gas molecules trapped during the production of packages. The sensors were attached to LCC packages using AuSn eutectic preform in a UniTemp RSS-160 reflow chamber. A 2-mm diameter circular $\mathrm{Cr} / \mathrm{Au}(500 \AA / 3000 \AA)$ was patterned on the backside of SOI dies for eutectic die attachment, [13]. The eutectic die attachment was performed at $375{ }^{\circ} \mathrm{C}$ for 20 minutes under $15 \mathrm{kPa}$ pressure applied to the bonding area. The process was optimized to obtain a void-free attachment, otherwise, the trapped air voids in the die attachment area would act as an outgassing source after vacuum sealing.

\section{Sealing Solder Preform Tack Welding}

The sorption capacity of the getter is directly related to the getter activation temperature. A higher relative pumping capacity can be reached at higher getter activation temperatures. The getter activation temperature is higher than the sealing solder eutectic point. To activate the getter at high temperatures without interfering with the sealing solder preforms, the preforms were tack-welded to the package seal ring using a low-power resistive welding with parallel electrodes, Fig. 2. For each tack-welding spot, 25 watts power was applied for 25 milliseconds. Fig. 3 shows a packaged device with solder preform attached to the package with three welding spots on each side using the tack-welding setup.

\section{Vacuum Sealing Process Development}

An SST 3150 high-vacuum furnace was utilized for vacuum sealing of MEMS sensors. This furnace is equipped with a turbomolecular drag pump and a cryogenic water pump and can reach a base pressure

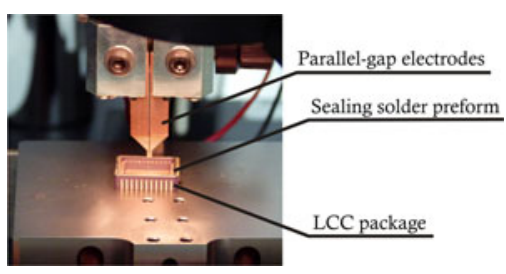

Fig. 2. Low power tack-welding setup to attach AuSn sealing solder preform frame to the seal ring of an LCC package.

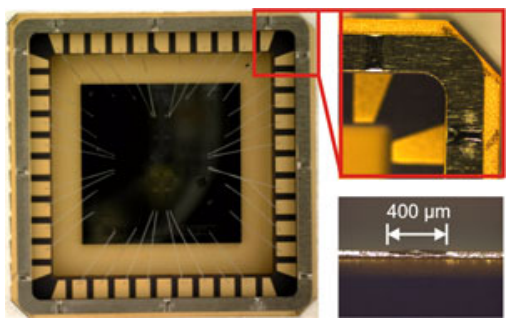

Fig. 3. LCC package with sealing solder preform attached using tackwelding (left), a magnified view of welding spots (top-right), a side view of a welding spot showing $\sim 400 \mu \mathrm{m}$ width of the heat affected zone.
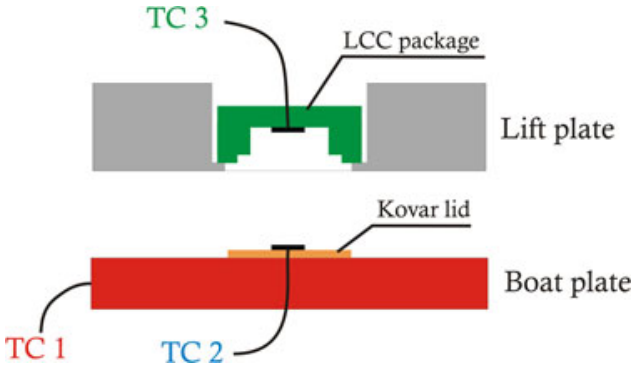

Fig. 4. Schematics of the graphite tooling assembly and temperature reading setup; additional thermocouples (TC) were used during the process development.

of $10^{-8}$ Torr. A customized graphite fixture assembly was used to accommodate LCC packages and Kovar lids. A schematic of the graphite fixture is illustrated in Fig. 4. Lids were placed on the bottom plate, i.e., "boat plate," with getter facing up. The boat plate temperature rises with the amount of current passing through the plate, the main thermocouple of the furnace is inserted into the boat plate for the thermal feedback control loop. LCC packages were loaded upside down on the top plate, i.e., "lift plate." Two additional thermocouples were attached to an LCC package and a Kovar lid for temperature monitoring of parts during the process development. A lift mechanism, inside the furnace, controls the separation between the boat plate and the lift plate.

Fig. 5 illustrates the separation of the boat and the lift plate at each step of the sealing process. During the pre-bake step, the lift plate was placed close to the boat plate. The temperature during the prebake step was adjusted to ensure effective desorption of gas and water molecules from the surfaces while avoiding a pre-mature activation of the getter. The target temperature for pre-bake was chosen to be $200{ }^{\circ} \mathrm{C}$, for a prolonged duration of more than 24 hours.

Fig. 6 illustrates the temperature profile of the sealing process accompanied by direct temperature readings from Kovar lid and LCC package. An in-situ getter activation followed the pre-bake step, before the lid sealing. The minimum getter activation temperature was $300{ }^{\circ} \mathrm{C}$. However, the activation of getter at higher temperature would 


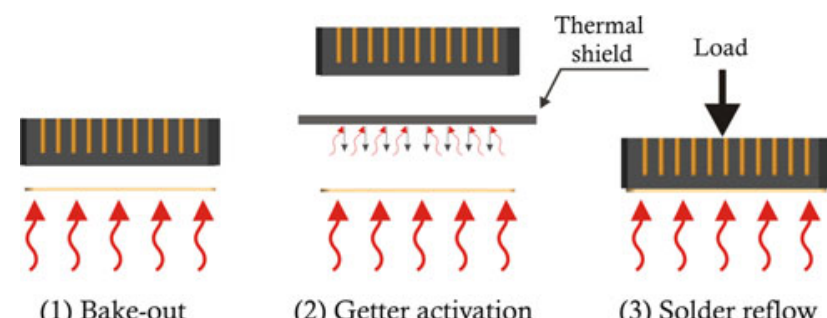

(1) Bake-out

(3) Solder reflow
Fig. 5. Configurations of lids and packages throughout the sealing process, with in-situ getter activation. (1) Packages are near the heat source during bake-out. (2) Thermal shield isolates the package and solder frame from overheating during getter activation. (3) Package is pressed against the lid during eutectic bonding.

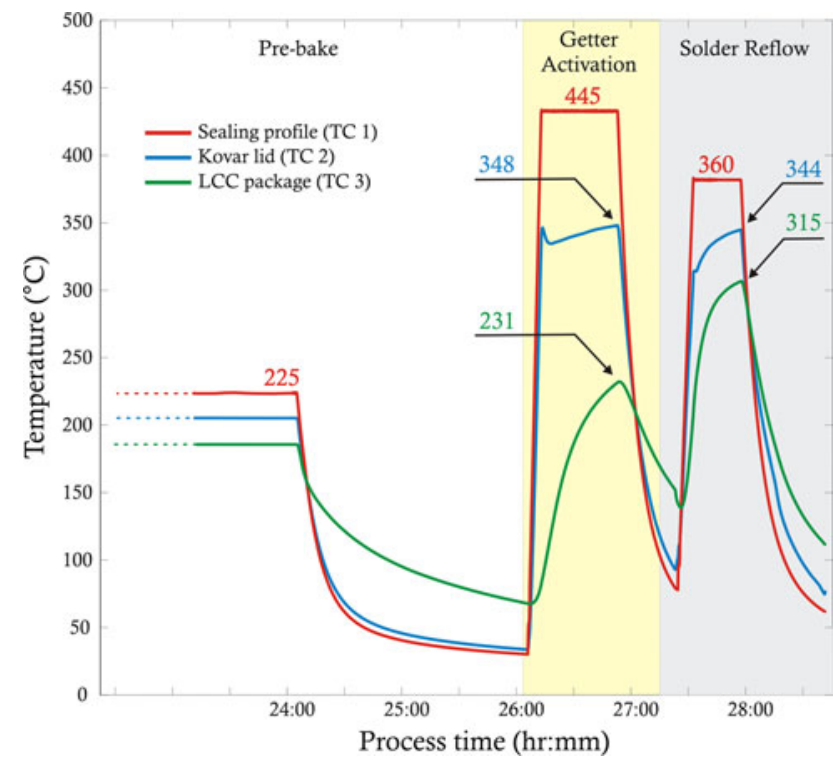

Fig. 6. Temperature profile of the sealing process (red) with simultaneous temperature recording from the package (green) and the lid (blue).

improve its gas sorption performance, [12]. Increasing the getter activation temperature may cause reflow of the sealing solder preform on the package before bringing it in contact with the lid. Thus, the temperature of the package during the getter activation should be maintained with a margin of safety below the reflow temperature of AuSn eutectic alloy, which is $278{ }^{\circ} \mathrm{C}$. Under the high-vacuum condition, thermal radiation is the main heat transfer mechanism. Radiation of heat from the boat plate would increase the temperature of the lift plate and could thermally damage the solder preform at higher temperatures. A shutter mechanism, integrated inside the furnace, thermally shields the packages from overheating. The thermal shielding, combined with tack-welding of solder preforms to the packages, protects the reflow of sealing solder preform and enables activation of the getter at a higher temperature. The getter was activated at $350{ }^{\circ} \mathrm{C}$ for 40 minutes. Fig. 7 shows the chamber base pressure readings at the getter activation and the sealing steps. The pressure spikes to $20 \mu$ Torr during the getter activation. After the getter activation, the chamber was cooled down by forced convection to reduce the base pressure to less than $0.1 \mu$ Torr before lid sealing.

The temperature profile of the solder reflow step was adjusted to achieve a defect-free eutectic bond between the lids and packages.

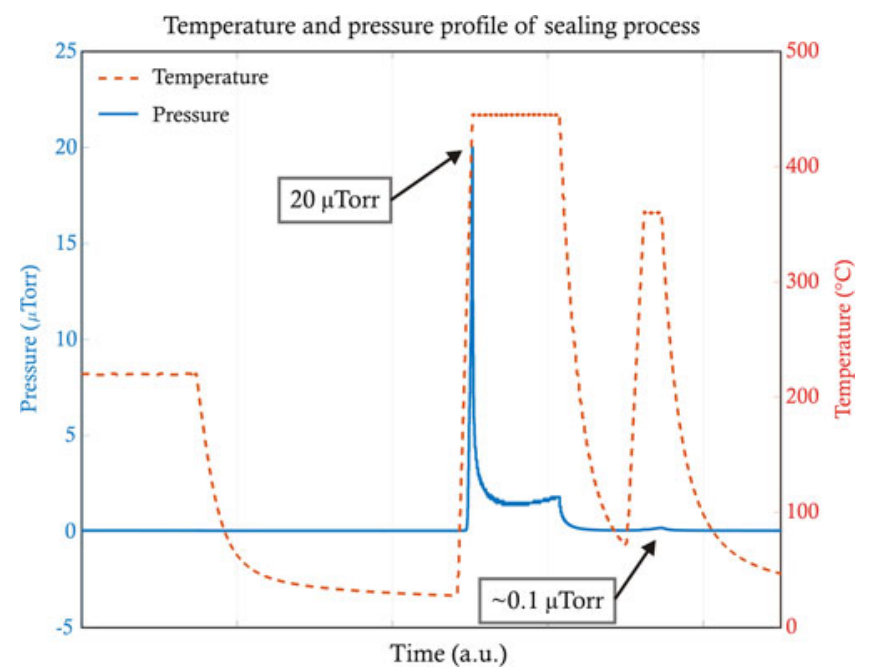

Fig. 7. Chamber base pressure of $20 \mu$ Torr and $0.1 \mu$ Torr at getter activation and lid sealing step, respectively.

Table 1. Vacuum Sealing Process Parameters (Using SST 3150).

\begin{tabular}{lccc}
\hline \hline Parameter & Pre-bake & Getter activation & Solder reflow \\
\hline Time & $24 \mathrm{hrs}$ & $40 \mathrm{~min}$ & $8 \mathrm{~min}$ \\
Temperature $\left({ }^{\circ} \mathrm{C}\right)$ & 225 & 445 & 360 \\
Temp. of lid $\left({ }^{\circ} \mathrm{C}\right)$ & 205 & 348 & 344 \\
Temp. of package $\left({ }^{\circ} \mathrm{C}\right)$ & 185 & 231 & 315 \\
\hline \hline
\end{tabular}

X-ray imaging was utilized to inspect the seal ring after vacuum sealing and detect any air voids. The void formation and growth was reduced by modifying the temperature-time profile of the pre-bake and lid sealing. Formation of many air voids in the sealing area indicates an insufficient pre-bake of package and lids. Excessive time and temperature of solder reflow step forms large and grown air voids, which eventually causes a leakage in the sealing area. The optimum time and temperature of the sealing step were adjusted in our process to fully reflow the eutectic solder frame with the minimal air void formation. The parameters of each step are summarized in Table 1.

It should be noted that devices undergo a thermal cycle during the sealing process. Indeed, the temperature compatibility of materials with the process temperature should be considered for adapting this process for other MEMS structures.

\section{EXPERIMENTAL RESULTS}

QMG sensors were wire bonded and characterized in the vacuum chamber before vacuum sealing. The Q-factor was measured using energy decay time at different vacuum levels and was used as a reference for vacuum assessment after sealing. The $\mathrm{Q}$-factor was monitored over a period of one year after vacuum sealing.

Fig. 8 shows the Q-factor evolution of two sensors sealed with different pre-bake durations. It was observed that the Q-factor after sealing reached over 2 million for sensor no. 1 with 24 hours prebaking, whereas the maximum Q-factor on the same sensor in the vacuum chamber was 0.5 million at 0.2 millitorr.

This results also revealed an improvement of the Q-factor over time in both cases, which indicates a continuous sorption of 


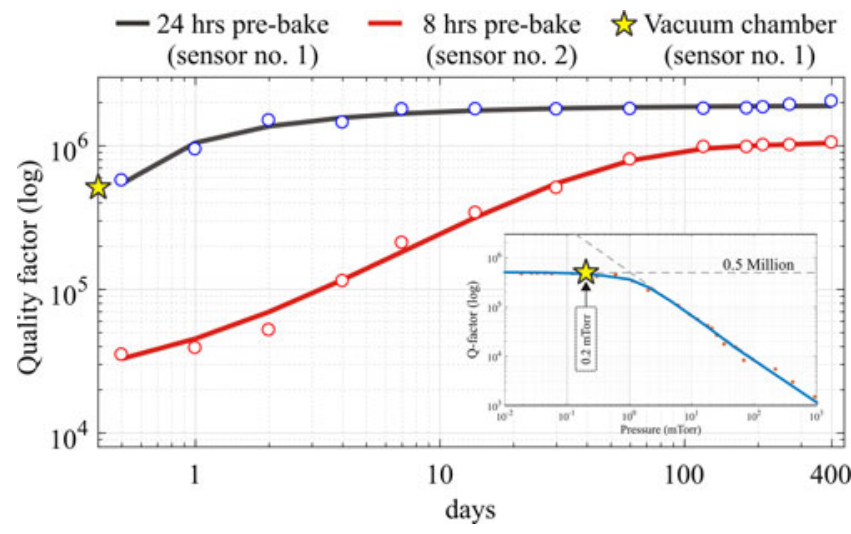

Fig. 8. Q-factor measurement over a period of 1 year after vacuum sealing of two devices with different pre-bake durations. (Inset) Q-factor of sensor no. 1 was measured in the vacuum chamber, showed maximum $Q$ of $\sim 0.5$ million at 0.2 millitorr.

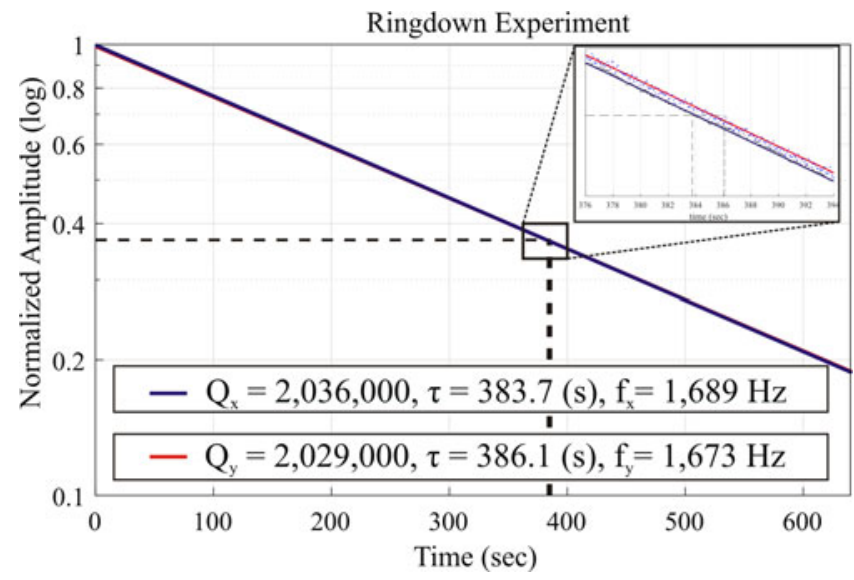

Fig. 9. Ringdown time measurement of a sealed QMG sensor one year after vacuum sealing, revealing a Q-factor higher than 2 million on both modes.

residual gas molecules by the activated getter. In addition, these results demonstrated that longer pre-baking reduces residual gas molecules inside the hermetically sealed package, enabling a more effective getter pumping and achieving a higher vacuum level after sealing. This phenomenon is discussed and numerically simulated in [12], showing a similar trend with the experimental results in Fig. 8. The results also showed a high Q-factor over the 1-year period, revealing a long-term stable ultra-high vacuum level after sealing.

Fig. 9 shows the ringdown time experiment of a sealed sensor, 1 year after sealing, demonstrated over 380 seconds of energy decay time, revealing over 2 million Q-factor, approaching the TED limit of the sensor structure (2.9 million from FEA for this design), thus demonstrating the effective elimination of air damping. In addition, the anti-phase motion of the dynamically-balanced QMG assures cancellation of reaction forces and moments on the anchors, reducing the energy loss through the substrate. These are the conditions required to reach the near-navigation grade performance of gyroscopes. Details of the tested device were published in [4]. The hermeticity of the seal was tested according to MIL-STD 883 method 1014. Sealed packages were bombarded by Helium (He) gas for 5 hours at 60 psig. The detected leak rate was $\sim 3.1 \times 10^{-8}(\mathrm{mbar} 1 / \mathrm{s})$ which was at the limit of the testing equipment. The Helium leak test results demonstrate the hermeticity of the seal.

\section{CONCLUSION}

We developed a package-level vacuum sealing process with getter activation for high-performance MEMS inertial sensors. The process parameters were adjusted to maximize de-sorption of gas molecules prior to the encapsulation, to efficiently activate getter, and to obtain a defect-free sealing. The integrated getter was activated and continuously pumped the residual and desorbed gases, improving the quality factor over a period of one year. The experimental results reported in this work confirmed the reliability and repeatability of the proposed vacuum sealing procedure. This paper suggests the suitable process parameters and requirements that can be adapted either for waferlevel high-vacuum packaging or other MEMS sensors application with ultra-high vacuum requirements.

\section{ACKNOWLEDGMENT}

The authors would like to acknowledge P. Zappella of SST International for the helium leak experiments. The design, characterization, and vacuum sealing of this work was done in UCI Microsystems Laboratory. Fabrication was done at UCI INRF facility.

This work was supported by DARPA under Contract no. N66001-12-C-4035.

\section{REFERENCES}

[1] M. Weinberg, R. Candler, S. Chandorkar, J. Varsanik, T. Kenny, and A. Duwel, "Energy loss in MEMS resonators and the impact on inertial and RF devices," presented at the Int. Conf. Solid-State Sensors, Actuators Microsyst., Denver, CO, USA, Jun. 2009.

[2] A. D. Challoner, H. G. Howard, and J. Y. Liu, "Boeing disc resonator gyroscope," presented at the Int. Symp. IEEE/ION Position, Location Navigation, Monterrey, CA, USA, May 2014.

[3] S. A. Zotov et al., "Investigation of energy dissipation in low frequency vibratory MEMS demonstrating a resonator with 25 minutes time constant," in presented at the Int. Conf. Solid-State Sensors, Actuators, Microsyst., Hilton Head, SC, USA, Jun. 2014.

[4] S. Askari, M. H. Asadian, K. Kakavand, and A. M. Shkel, "Near-navigation grade quad mass gyroscope with Q-factor limited by thermo-elastic damping," in presented at the Int. Conf. Solid-State Sensors, Actuators, Microsyst., Hilton Head, SC, USA, Jun. 2016.

[5] T. Nagourney, J. Y. Cho, B. Shiari, A. Darvishian, and K. Najafi, " 259 second ringdown time and 4.45 million quality factor in $5.5 \mathrm{kHz}$ fused silica birdbath shell resonator," presented at the Int. Conf. Solid-State Sensors, Actuators Microsyst., Kaohsiung, Taiwan, Jun. 2017.

[6] D. Senkal, M. J. Ahamed, M. H. A. Ardakani, S. Askari, and A. M. Shkel, "Demonstration of 1 million Q-factor on microglassblown wineglass resonators with outof-plane electrostatic transduction," J. Microelectromech. Syst., vol. 24, pp. 29-37, 2015.

[7] A. B. Graham et al., "A method for wafer-scale encapsulation of large lateral deflection MEMS devices," J. Microelectromech. Syst., vol. 19, pp. 28-37, 2010.

[8] S. H. Lee, J. Cho, S. W. Lee, M. F. Zaman, F. Ayazi, and K. Najafi, "A lowpower oven-controlled vacuum package technology for high-performance MEMS," presented at the 22nd Int. Conf. IEEE Micro Electro Mechanical Syst., Sorrento, Italy, Jan. 2009

[9] M. Esashi, "Wafer level packaging of MEMS," J. Micromech. Microeng., vol. 18, no. 7, 2008, Art. no. 073001 .

[10] S. H. Choa, "Reliability of MEMS packaging: Vacuum maintenance and packaging induced stress," Microsyst. Technol., vol. 11, pp. 1187-1196, 2005.

[11] T. F. Marinis and J. W. Soucy, "Vacuum packaging of MEMS inertial sensors," in Proc. Int. Symp. Microelectron., Boston, MA, USA, Nov. 2003.

[12] E. Rizzi et al., "Outgassing and gettering," in Handbook of Silicon Based MEMS Materials and Technologies, 2nd ed, M. Tilli, T. Motooka, V. M. Airaksinen, S. Franssila, M. Paulasto-Krockel, and V. Lindroos, Eds. New York, NY, USA: Elsevier, 2015, pp. 713-733.

[13] I. P. Prikhodko, B. R. Simon, G. Sharma, G. A. Zotov, A. A. Trusov, and A. M Shkel, "High and moderate-level vacuum packaging of vibratory MEMS," in Proc 46th Int. Conf. Microelectron., Orlando, FL, USA, Oct. 2013. 